\title{
Pain catastrophizing as a risk factor for chronic pain after total knee arthroplasty: a systematic review
}

This article was published in the following Dove Press journal:

Journal of Pain Research

5 January 2015

Number of times this article has been viewed

\author{
Lindsay C Burns ${ }^{1-3}$ \\ Sarah E Ritvo' \\ Meaghan K Ferguson' \\ Hance Clarke ${ }^{3-5}$ \\ Ze'ev Seltzer ${ }^{3,5}$ \\ Joel Katz ${ }^{1,3-5}$ \\ 'Department of Psychology, York \\ University, Toronto, ON, Canada; \\ ${ }^{2}$ Arthritis Research Centre of Canada, \\ Vancouver, BC, Canada; ${ }^{3}$ Department \\ of Anesthesia and Pain Management, \\ Toronto General Hospital, Toronto, \\ ON, Canada; ${ }^{4}$ Department of \\ Anesthesia, University of Toronto, \\ Toronto, ON, Canada; ${ }^{5}$ Centre for the \\ Study of Pain, Faculties of Dentistry \\ and Medicine, University of Toronto, \\ Toronto, ON, Canada
}

\section{Video abstract}

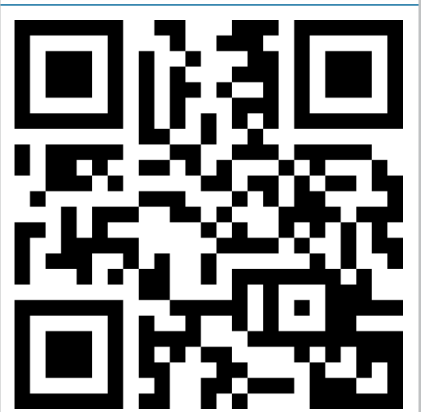

Point your SmartPhone at the code above. If you have a QR code reader the video abstract will appear. Or use: http://dvpr.es/ItVLK6W

Correspondence: Lindsay C Burns; Joel Katz

Department of Psychology, Behavioral Science Building, York University, 4700 Keele Street, Toronto,

ON M3J IP3, Canada

Email Icburns@yorku.ca; jkatz@yorku.ca
Background: Total knee arthroplasty (TKA) is a common and costly surgical procedure. Despite high success rates, many TKA patients develop chronic pain in the months and years following surgery, constituting a public health burden. Pain catastrophizing is a construct that reflects anxious preoccupation with pain, inability to inhibit pain-related fears, amplification of the significance of pain vis-à-vis health implications, and a sense of helplessness regarding pain. Recent research suggests that it may be an important risk factor for untoward TKA outcomes. To clarify this impact, we systematically reviewed the literature to date on pain catastrophizing as a prospective predictor of chronic pain following TKA.

Methods: We searched MEDLINE, EMBASE, and PsycINFO databases to identify articles related to pain catastrophizing, TKA, risk models, and chronic pain. We reviewed titles and abstracts to identify original research articles that met our specified inclusion criteria. Included articles were then rated for methodological quality. including methodological quality. Due to heterogeneity in follow-up, analyses, and outcomes reported across studies, a quantitative metaanalysis could not be performed.

Results: We identified six prospective longitudinal studies with small-to-mid-sized samples that met the inclusion criteria. Despite considerable variability in reported pain outcomes, pain catastrophizing was identified as a significant predictor of chronic pain persisting $\geq 3$ months following TKA in five of the studies assessed. Limitations of studies included lack of largescale data, absence of standardized pain measurements, inadequate multivariate adjustment, such as failure to control for analgesic use and other relevant covariates, and failure to report non-significant parameter estimates.

Conclusion: This study provides moderate-level evidence for pain catastrophizing as an independent predictor of chronic pain post-TKA. Directions for future research include larger, well-controlled studies with standard pain outcomes, identification of clinically-relevant catastrophizing cut-offs that predict pain outcomes, investigation of other psychosocial risk factors, and assessment of interventions aimed to reduce pain catastrophizing on chronic pain outcomes following TKA surgery.

Keywords: pain catastrophizing, total knee arthroplasty, total knee replacement, knee arthroplasty, risk factors, chronic pain

\section{Significance}

1. A moderate level of evidence was obtained for pain catastrophizing as an independent predictor of chronic post-surgical pain following total knee arthroplasty (TKA);

2. Lack of uniformity in data capture, particularly in pain outcome measures, precludes meta-analysis and underscores the need for consensus regarding standardized reporting of chronic pain outcomes; 
3. Future directions for research include larger study samples, improved covariate adjustment (especially for analgesic use, depression, and anxiety), adoption of consensus guidelines on outcome measures for clinically relevant chronic pain, optimal thresholds for pain catastrophizing levels that predict adverse pain outcomes, and assessment of interventions aimed to reduce the negative effects of pain catastrophizing symptoms on chronic pain outcomes.

\section{Introduction}

With the aging population and mounting obesity epidemic, rates of TKA have increased dramatically in the last two decades such that TKA now constitutes one of the most common and costly medical procedures in the US and Canada. ${ }^{1-3}$ Although studies employing survivorship and surgeon-based measures have generally reported TKA success rates exceeding $80 \%,{ }^{4}$ these measures do not account for post-surgical pain following TKA such that chronic pain remains a major health burden for many patients. ${ }^{5}$ Specifically, studies utilizing patient-based outcome measures have indicated that $6 \%-30 \%$ of patients continue to experience chronic pain in the months and years after TKA. ${ }^{6-10}$ This critical gap in recovery outcomes has prompted investigators to search for modifiable risk factors for chronic pain outcomes after TKA surgery.

Converging evidence from the literature on non-surgical chronic pain suggests that pain disability does not result solely from the severity of the pain, but rather, is largely influenced by patients' interpretation and adjustment to their pain. ${ }^{11-13} \mathrm{In}$ particular, pain catastrophizing has emerged as an important factor in chronic pain onset, severity, and duration, and may represent an independent predictor of poor treatment outcomes including the development of chronic pain after surgery. ${ }^{14}$ Pain catastrophizing is a multidimensional construct comprising elements of rumination (ie, an anxious preoccupation with pain and the inability to inhibit pain-related thoughts and fears), magnification (ie, the tendency to amplify the significance of pain with respect to implications for one's global health), and helplessness (ie, despair surrounding perceived inability to control one's pain experience). ${ }^{15}$ Accordingly, high levels of pain catastrophizing have been shown to predict high levels of acute and persistent pain after various surgeries. ${ }^{16,17}$ Further, a recent systematic review of psychological risk factors for pain in total knee and hip arthroplasty included catastrophizing as a potential risk factor, although the search strategy employed was somewhat limited leading to the omission of key studies. ${ }^{18}$

Although prospective studies have emphasized the role of pain catastrophizing as a risk factor for poor pain outcomes following surgical procedures including TKA, ${ }^{19}$ the majority of research has employed the outcome of acute pain (ie, $<3$ months following surgery). ${ }^{20}$ Whereas acute pain tends to be proportionate to the degree of tissue damage and is generally functional, pain that persists for $\geq 3$ months duration is considered chronic given that such pain exceeds typical physical healing time and often constitutes its own disorder that perpetuates functional disability. ${ }^{21}$ However, the association between pain catastrophizing and chronic pain outcomes remains to be specifically and systematically analyzed in TKA populations. Thus, to clarify our understanding of a potentially modifiable psychological risk factor for a major surgical complication, we aimed to systematically review the literature that reported the prospective impact of pain catastrophizing on pain that persists $\geq 3$ months after TKA.

\section{Methods}

\section{Search strategy}

We conducted a literature search of MEDLINE (January 1, 1946-August 25, 2014), EMBASE (January 1, 1980-August 25, 2014), and PsycINFO (January 1, 1872-August 25, 2014) databases. Our search strategy employed mapped medical subject headings (MeSH) terms combined with keywords for unindexed terms related to the themes of pain catastrophizing, TKA, risk factors, and chronic pain (see Table 1 for detailed

Table I Mapped medical subject headings (MeSH) terms and keywords employed in electronic search strategy

\begin{tabular}{|c|c|c|}
\hline Concept & MeSH terms & Keywords \\
\hline Catastrophizing & $\begin{array}{l}\text { Catastrophization; behavioral } \\
\text { symptoms; behavioral medicine; } \\
\text { stress, psychological; health behavior; } \\
\text { psychology, social; mental disorders; } \\
\text { anxiety; anxiety disorders; neurotic } \\
\text { disorders; personality; attitude to } \\
\text { health }\end{array}$ & $\begin{array}{l}\text { Catastrophiz*; } \\
\text { emotional; } \\
\text { attitud*; } \\
\text { coping; pain } \\
\text { catastrophizing } \\
\text { scale; PCS }\end{array}$ \\
\hline $\begin{array}{l}\text { Total knee } \\
\text { arthroplasty }\end{array}$ & $\begin{array}{l}\text { General surgery; postoperative } \\
\text { complications; total knee } \\
\text { replacement }\end{array}$ & $\begin{array}{l}\text { Surgery; } \\
\text { post-surg*; } \\
\text { postsurg*; } \\
\text { post-operati*; } \\
\text { postoperati*; } \\
\text { TKA; TKR }\end{array}$ \\
\hline Risk factors & $\begin{array}{l}\text { Risk; proportional hazards models; } \\
\text { regression analysis; logistic models; } \\
\text { risk factors; models, statistical; } \\
\text { survival analysis; epidemiologic } \\
\text { research design; epidemiology; } \\
\text { epidemiologic methods; } \\
\text { epidemiologic studies; models, } \\
\text { psychological }\end{array}$ & $\begin{array}{l}\text { Risk factor*; } \\
\text { predict* }\end{array}$ \\
\hline Chronic pain & $\begin{array}{l}\text { Pain; postoperative; chronic } \\
\text { pain; pain, intractable }\end{array}$ & Pain \\
\hline
\end{tabular}

Abbreviations: PCS, Pain Catastrophizing Scale; TKA, total knee arthroplasty; TKR, total knee replacement. 
search strategy). Additional articles were identified through a manual search of relevant bibliographies.

\section{Study selection}

After removal of duplicates, two authors (LB, SR) independently reviewed titles and abstracts to identify published studies that met our systematic review inclusion criteria of: 1) pain catastrophizing exposure; 2) TKA patient population; 3 ) chronic pain outcome (ie, assessed $\geq 3$ months postoperatively); 4) prospective and longitudinal study design (eg, cohort, case-control studies with measurement of exposure prior to outcome); 5) original research paper; and 6) English language. We excluded non-primary literature (eg, reviews and commentaries), non-peer reviewed studies (eg, graduate theses), and conference proceedings. Any discrepancies in the selection of articles were resolved via consensus. After a manual search of relevant bibliographies to identify additional articles, abstracts that met our inclusion criteria were forwarded for full-text review. Two authors (LB and SR) independently assessed full-text articles for inclusion on the basis of the eligibility criteria, and any discrepancies were resolved via consensus.

\section{Quality assessment}

To assess the methodological quality of included studies, we adapted a checklist based on an established quality assessment instrument from the orthopedic literature. ${ }^{22,23}$ Two reviewers (LB and $\mathrm{JK}$ ) independently scored the included studies according to 14 quality criteria (Table 2). Each criterion that was met was assigned a score of 1 and each that was not (or with insufficient information provided to assess) was assigned a score of 0 . Any discrepancies were resolved via consensus. The maximum potential score for cohort designs (ie, study groups assigned based on exposure) was 14 and the maximum potential score for case-control designs (ie, study groups assigned based on outcome) was 13 . The quality rating for each study was reported as the percentage of obtained scores out of the maximum potential score. ${ }^{22}$

\section{Data abstraction and synthesis}

We developed standardized data abstraction forms for uniform data capture across raters. Two authors (LB and SR) abstracted the data for each article and another (JK) verified the accuracy of the abstracted data. For each included study, we obtained the following information: citation, country,

Table 2 Quality assessment of studies included in systematic review

\begin{tabular}{|c|c|c|c|c|c|c|}
\hline & $\begin{array}{l}\text { Edwards } \\
\text { et } \mathrm{al}^{5} \text {, } \\
2009 \text { [SI] }\end{array}$ & $\begin{array}{l}\text { Forsythe } \\
\text { et } \mathrm{al}^{24} \\
2008 \text { [S2] }\end{array}$ & $\begin{array}{l}\text { Masselin-Dubois } \\
\text { et } \mathrm{al}^{25} \\
2013 \text { [S3] }\end{array}$ & $\begin{array}{l}\text { Noiseux } \\
\text { et al }{ }^{26} \text {, } \\
20 \mid 4[54]\end{array}$ & $\begin{array}{l}\text { Riddle } \\
\text { et } \mathrm{al}^{27} \\
2010 \text { [S5] }\end{array}$ & $\begin{array}{l}\text { Sullivan } \\
\text { et } \mathrm{al}^{28} \text {, } \\
201 \mathrm{I} \text { [S6] }\end{array}$ \\
\hline QI-Clear description of selection of study subjects & I & $\mathrm{I}$ & I & I & 0 & $\mathrm{I}$ \\
\hline Q2-Formal power/sample size calculation & 0 & 0 & 0 & 0 & $\mathrm{I}$ & 0 \\
\hline $\begin{array}{l}\text { Q3-Number of subjects assessed for eligibility vs } \\
\text { enrolled adequately described }\end{array}$ & 0 & 1 & 1 & 1 & I & 0 \\
\hline Q4-Sufficient description of characteristics of subjects & I & $\mathrm{I}$ & $\mathrm{I}$ & $\mathrm{I}$ & 0 & $\mathrm{I}$ \\
\hline Q5-Participation rate $\geq 80 \%$ for study groups & 0 & 0 & 0 & 0 & 0 & 0 \\
\hline Q6-Attrition documented & 0 & $\mathrm{I}$ & $\mathrm{I}$ & $\mathrm{I}$ & I & 0 \\
\hline $\begin{array}{l}\text { Q7-Pain catastrophizing exposure measured } \\
\text { with valid and reliable tool }\end{array}$ & I & I & 1 & 1 & I & I \\
\hline $\begin{array}{l}\text { Q8-Pain outcome(s) measured with valid } \\
\text { and reliable tool }\end{array}$ & 0 & I & I & I & I & I \\
\hline $\begin{array}{l}\text { Q9-Distribution of comorbidities/covariates } \\
\text { provided for study groups }\end{array}$ & I & $N / A$ & I & $N / A$ & $\mathrm{~N} / \mathrm{A}$ & I \\
\hline $\begin{array}{l}\text { QI0-Comorbidity/covariates measured } \\
\text { identically in study groups }\end{array}$ & I & I & I & I & I & I \\
\hline $\begin{array}{l}\text { QI I-Results adjusted for potential confounders } \\
\text { (minimally: age, sex, and pre-op pain rating) }\end{array}$ & I & 0 & I & I & I & I \\
\hline $\begin{array}{l}\text { Q I2-Results adjusted for psychosocial factors } \\
\text { (minimally: anxiety and depression) }\end{array}$ & 0.5 & 0 & I & I & I & 0.5 \\
\hline QI3-Appropriate analysis techniques used & 1 & 0 & 1 & $\mathrm{I}$ & 1 & 1 \\
\hline QI4-Sufficient info given on association (effect) sizes & I & I & I & I & I & I \\
\hline Obtained score & 8.5 & 8.5 & 12 & II & 10 & 9.5 \\
\hline Potential score & 14 & 13 & 14 & 13 & 13 & 14 \\
\hline Quality rating, \% (obtained / potential score $\times 100$ ) & 61 & 65 & 86 & 85 & 77 & 68 \\
\hline
\end{tabular}

Note: N/A, not applicable (presentation of covariate distribution across study [SI-S6] groups is not appropriate in case-control studies, since baseline covariates are confounded by outcome). 
patient population, sample size, age, sex, body mass index (BMI), study design, follow-up time, exposure (pain catastrophizing) definition, outcome (pain) definition, analysis type, adjusted covariates/confounders, and main results. Due to the heterogeneity in reported pain outcomes, statistical analyses, and follow-up periods across identified studies, a quantitative meta-analysis could not be performed.

\section{Results}

\section{Literature search results}

We identified 17,425 articles after the removal of duplicates (Figure 1). Following title and abstract review, 23 fulltext articles were assessed and excluded for the following reasons: no pain catastrophizing exposure $(n=2)$; no chronic pain ( $\geq 3$ months after surgery) outcome $(n=2)$; no TKA population/no TKA-specific results $(n=5)$; and incorrect study type (eg, reviews, commentaries; $n=14$ ). Overall, six studies met our eligibility criteria. Characteristics and quality ratings of included studies are summarized in Table 2.

\section{Overview of studies}

We identified six studies ${ }^{5,24-28}$ (S1-S6 in Tables 3 and 4) that assessed the impact of pain catastrophizing on chronic postsurgical TKA pain. Details of studies are summarized in Table 3 and extracted results are presented in Table 4. Sample sizes ranged from 55 to 215 participants and females constituted

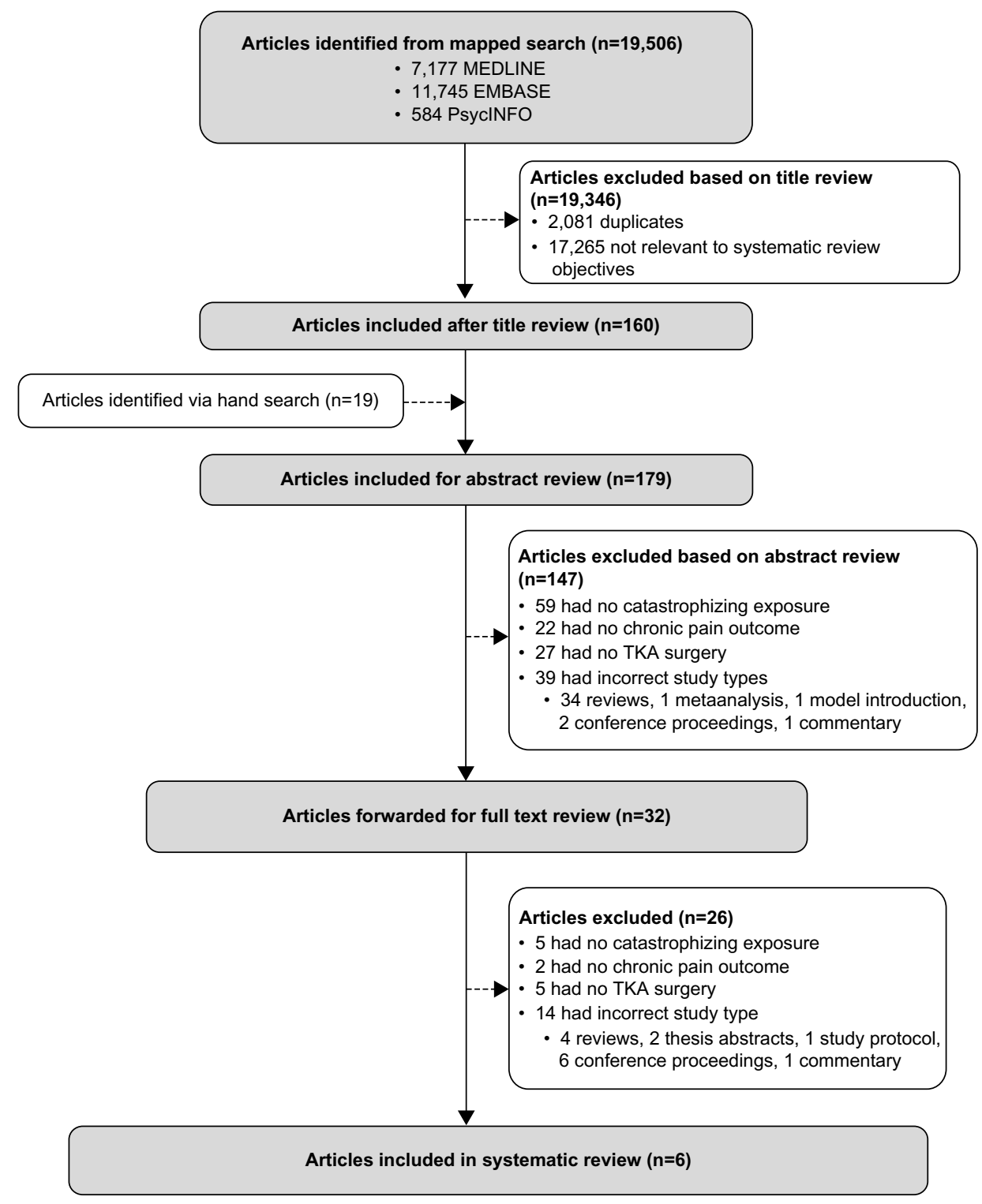

Figure I Flow chart showing numbers of studies screened, assessed for eligibility, and included in the present review. Also shown are reasons for exclusions at each stage and numbers of articles excluded.

Abbreviation: TKA, total knee arthroplasty. 


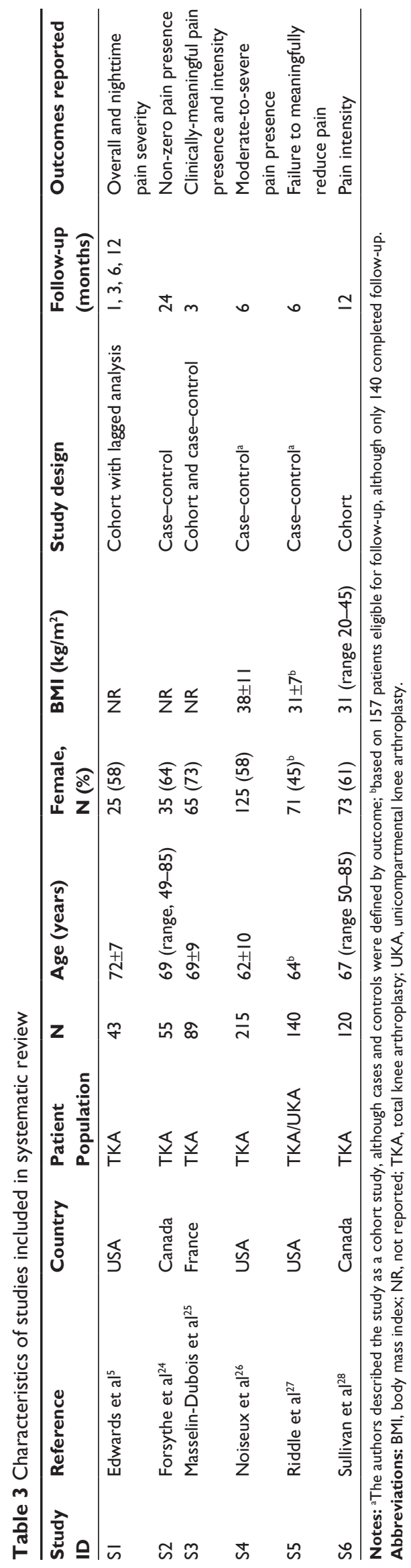

between $45 \%$ and $73 \%$ of study samples. Among studies that assessed BMI, patients tended to fall in the obese I/II range. Length of follow-up ranged from 3 months to 24 months. All studies measured the pain catastrophizing exposure preoperatively and employed reliable and valid assessment tools (five studies employed the pain catastrophizing scale ${ }^{15}$ and one used the catastrophizing subscale of the Coping Strategies Questionnaire. ${ }^{29}$ Study designs included cohort, case-control, and lagged analyses, and considerable variability was observed with respect to outcome definitions for chronic pain. Studies measured the main outcome of pain using a variety of instruments, including the ShortForm McGill Pain Questionnaire (SF-MPQ), ${ }^{30}$ Brief Pain Inventory (BPI) ${ }^{31}$ Western Ontario and McMaster University Osteoarthritis Index (WOMAC) pain score, ${ }^{32}$ and a variety of pain rating scales (eg, $10 \mathrm{~cm}$ visual analog scale [VAS], 21-point numerical rating scale [NRS], 0-100 scale). ${ }^{33}$ Pain intensity/severity was assessed with the patient resting, in response to range-of-motion exercises, thermal testing, pressure algometry, as a daily global score, and as nighttime pain. Moreover, pain was reported as an absolute value, a change from baseline, and/or as the number/percent of patients reporting pain scores above or below a certain threshold. The methodologic quality of studies was generally acceptable (Table 2), although one study did not control for confounding. Key evidence is synthesized below with consideration given to type of study type, exposure, and outcome measurements used, and follow-up time.

\section{Cohort studies}

We identified two prospective cohort studies (S3 and S6) from France and Canada that reported the impact of preoperative pain catastrophizing on chronic pain following TKA surgery. In both cases, multivariable linear regression was used with adjustment for relevant confounders including baseline age, sex, preoperative pain, and medical/ psychological covariates (S3 for comorbidities, anxiety, and depression; S6 for fear of movement and depression). Pain medication/analgesic use was not reported or controlled for in either case. Both studies measured the catastrophizing exposure using the Pain Catastrophizing Scale (PCS) and pain intensity was measured as a continuous outcome variable (S3, BPI; S6, WOMAC pain scale). In S3, increased levels of magnification (PCS-M subscore) independently predicted elevated pain intensity among 89 TKA patients ( $100 \%$ follow-up rate) at 3 months $(\beta=0.14, S E=0.05 ; t=2.06 ; P=0.04$; Table 4). Similarly, S6 found that heightened catastrophizing (overall score) 
independently predicted increased pain ratings among 120 TKA patients 1 year postoperatively, $(\beta=0.27, P<0.05$; Table 4). Further, they showed that behavioral outcome expectancies measured before surgery partially mediated the relationship between pain catastrophizing and 6-month follow-up WOMAC pain scores, which provides some evidence for potential intervention targets to help reduce chronic pain after TKA. Because the authors did not report on loss to follow-up, the impact of this potential bias cannot be determined.

Though not a traditional cohort study, we identified one additional study that used prospectively collected pain catastrophizing scores to predict subsequent pain intensity ratings as a continuous outcome variable $(\mathrm{S} 1)$. This small ( $n=43)$ US study (2009) employed generalized estimating equations (GEE) to conduct a lagged analysis with repeated measures of pain catastrophizing and pain severity at baseline (preoperatively) and 1 month, 3 months, 6 months, and 12 months follow-up. The authors leveraged the power of GEE to compensate for their small sample size and incomplete data capture over follow-up by allowing pain outcomes to be predicted by a pain catastrophizing score at any previous time point. The authors measured catastrophizing using the Coping Strategies Questionnaire-Catastrophizing Subscale (all other studies employed the PCS) and pain ratings were measured using a 0-100 scale. Multivariate adjustment was made for time point, pain, and depression; due to the repeated-measures design, demographic factors were also inherently controlled. Analgesic or other pain medication use was not reported. Before adding depression to their multivariable model, global daily (past $24 \mathrm{~h}$ ) and nighttime pain severity at each time point were positively related to pain catastrophizing levels at the previous time point. However, when depression was added to the daily global pain model, the effect of pain catastrophizing lost significance and only pain at the previous point and depression contributed significantly to the explanation of global daily pain. In contrast, after depression was added to the nighttime pain model, pain catastrophizing remained significant (global pain estimate $=2.1$; $S E=2.2 ; t=0.9 ; P=0.35$; nighttime pain estimate $=5.1 ; S E$ $=2.5 ; t=2.0 ; P=0.04$; Table 4$)$, although its contribution was reduced. These results suggest some degree of collinearity between depression and pain catastrophizing. Despite overall decreases in pain levels over time, pain catastrophizing levels did not vary significantly from baseline to the end of follow-up (Table 4). Together, these findings suggest that catastrophizing may constitute a stable driver of future pain severity in the year following TKA. It should be noted that the initial follow-up period (baseline to $1 \mathrm{month}$ ) violated our inclusion criterion of $\geq 3$ months follow-up for chronic pain and could thus lead to some contamination of results; however, we retained this study for evidence synthesis given that the majority of the model variance was derived from the other assessment time points, by which time the pain was longstanding enough to fit with our definition of chronic. Nonetheless, the higher proportion of individuals with pain at 1 month follow-up may have led to a slight overestimation of the impact of pain catastrophizing on subsequent pain variability.

\section{Case-control studies}

Four studies (S2, S3, S4, and S5) assessed the impact of pain catastrophizing according to several dichotomized outcome definitions of chronic pain, which differed across all included studies. Among these, two studies (one from the US [S4, $n=215]$ and one from France [S3; n=89]) used multivariate logistic regression to assess whether higher pain catastrophizing (PCS) scores increased the odds of clinically-relevant pain levels persisting after TKA surgery. The US case-control study was nested within an existing randomized clinical trial (RCT) of transcutaneous electrical nerve stimulation (TENS). This study (S4) defined "cases" as those with "moderate-tosevere pain intensity" (ie, a score $\geq 8$ on a 21 -point NRS for pain), whereas the latter (S3) defined "cases" as those with a "clinically-meaningful" pain intensity (ie, a score of $\geq 3 / 10$ on the BPI). Both studies adjusted for relevant baseline covariates including age, sex, pain, depression, and anxiety, and S4 additionally adjusted for medical comorbidities, fear of movement/re-injury, and RCT treatment arm. In both cases, pain catastrophizing was not found to be an independent predictor of clinically relevant pain levels at follow-up $(P>0.05$; Table 4). Neither of these studies reported odds ratios (ORs) with confidence intervals (CIs) for the TKA patients, masking potential trends and precluding future meta-analysis. Further, pre-operative anxiety emerged as an important predictor in both final models; however, the authors did not publish univariate or stepwise results from their logistic models. Thus, it remains unclear whether pain catastrophizing would have conferred a significant risk before depression and anxiety were added as covariates, which could again indicate collinearity of these constructs.

Another small scale study (S2; $=55)$ assessed the impact of pain catastrophizing on a dichotomized definition of pain defining the presence of non-zero pain at 24 months follow-up as the primary outcome. The authors used two instruments to assess their outcome of non-zero pain: 1) the Short-Form 


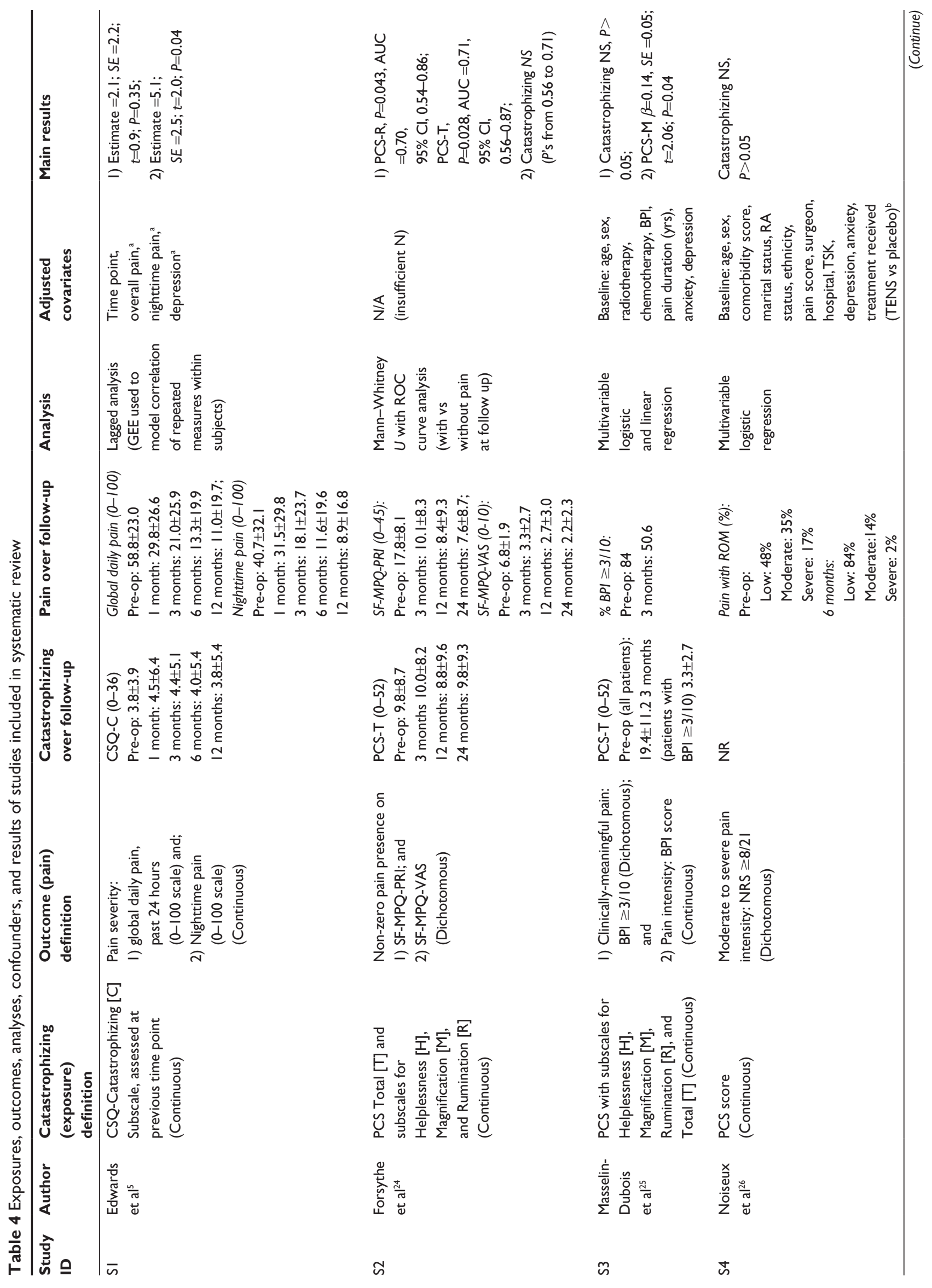




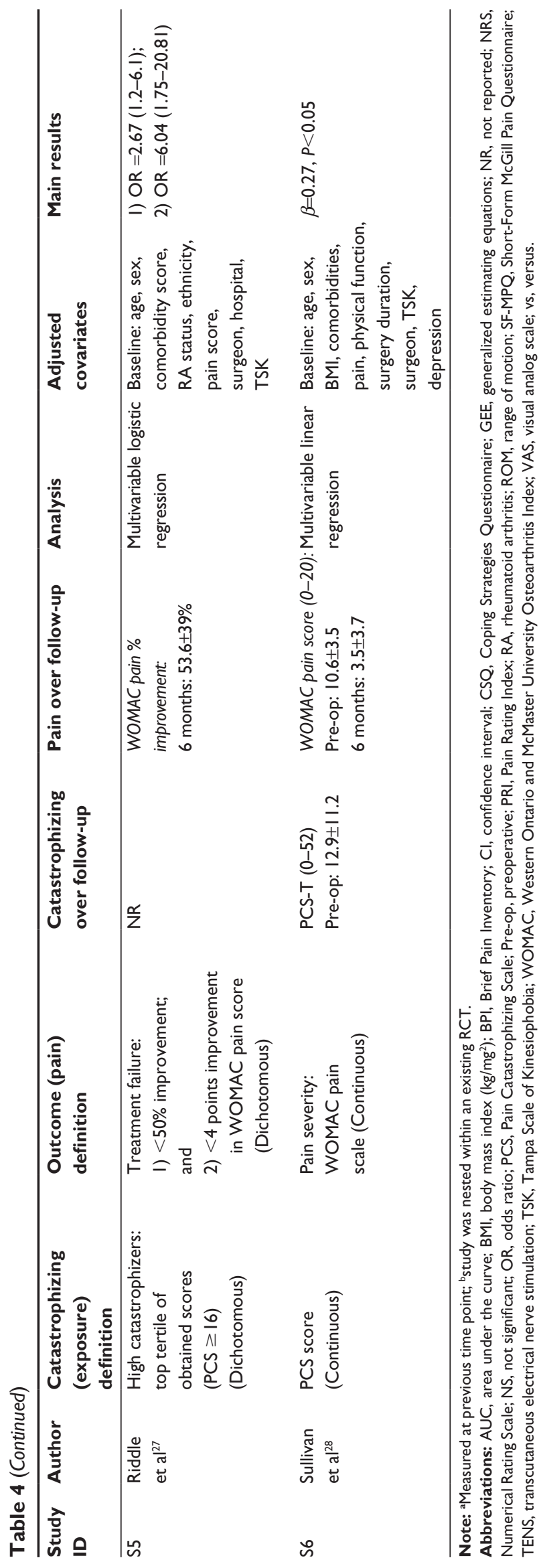

MPQ-Pain Rating Index (PRI), which asks participants to rate adjectives that describe qualitative aspects of pain on a 4-point scale, and 2) the MPQ-VAS. Because the data were skewed, the authors employed Mann-Whitney $U$ tests and receiver operating characteristic (ROC) curve analysis to assess the impact of catastrophizing on non-zero pain presence. The model using the adjective-based measure of non-zero pain (ie, MPQ-PRI), showed that both the total PCS score (PCS-T) and the rumination subscore (PCS-R) were significant predictors of the outcome (PCS-T, $P=0.028$, area under the curve $=0.71$; PCS-R, $P=0.043$, area under the curve $=0.70$ ). When the MPQ-VAS score was employed, however, the effect of catastrophizing lost significance ( $P$ 's from 0.56 to 0.71 ; Table 4). It is likely that the MPQ-PRI constitutes a more sensitive measure of variation among individuals with low-level pain because it prompts participants to consider different aspects of their pain experience, of importance in studies with extended follow-up intervals where floor effects can make relations difficult to detect. Nonetheless, caution should be exercised when interpreting these results given the small sample size, non-parametric analyses, failure to adjust for any covariates, and failure to adjust for multiple analyses, all of which increase the likelihood of false-positive findings.

Finally, we identified a mid-sized ( $\mathrm{n}=140$ ) US case-control study (S5) that used TKA treatment failure (insufficient pain reduction) at 6 months follow-up as the case definition. The authors also used the PCS to measure preoperative pain catastrophizing, but chose to dichotomize this predictor variable according to high vs low catastrophizers. High catastrophizers were arbitrarily defined as those individuals in the highest tertile of obtained scores, corresponding to PCS scores $\geq 16$, and all others were designated as low catastrophizers. It is noteworthy that the PCS manual provides cut-off scores for problematic pain catastrophizing; the selected threshold of $\geq 16$ constitutes the cut-off score for the 41 st percentile. ${ }^{34}$ Treatment failure was operationalized both in terms of 1) $<50 \%$ improvement in WOMAC pain scores from baseline; and 2) $<4$ point improvement in WOMAC pain scores (based on sensitivity analysis of patient-defined clinical relevance). After adjustment for covariates including baseline age, sex, pain, comorbidities, and fear of movement, the authors found that high catastrophizers had substantially increased odds of treatment failure according to $<50 \%$ WOMAC pain improvement $(\mathrm{OR}=2.67)$ and $<4$ point WOMAC pain improvement ( $\mathrm{OR}=6.04$ ) (Table 4). The authors did not adjust for depression, anxiety, or analgesic use, which could potentially covary with pain catastrophizing or confound the 
associations under investigation. Furthermore, the authors noted that of 157 individuals assessed at baseline, 129 underwent TKA, whereas 28 underwent unicompartmental (or partial) knee arthroplasty (UKA), which is a simpler operation indicated among individuals with lower disease severity. Only 140 patients completed follow-up assessments and were ultimately included in the case-control analysis, although the authors did not report on the composition of TKA vs UKA participants included in the final sample. It should be noted that inclusion of UKA individuals in the study population is a technical violation of our inclusion criteria; however, we retained the study in the systematic review because the majority of the variability would derive from the TKA patients. Although it is not possible to assess the specific degree of contamination conferred by UKA patients to the results, these individuals would likely have less intense overall pain (and less variability in pain) at followup, and thus, their inclusion would likely bias estimates toward the null should they have any impact. The authors did not conduct a sensitivity analysis to assess the impact of using alternate cut-offs for high catastrophizers, which would be of high clinical relevance for screening purposes.

\section{Impact of follow-up length}

Follow-up of included studies ranged from 3 months to 24 months. Overall, there was no clear indication that length of follow-up modified the relationship between preoperative pain catastrophizing and chronic pain outcomes during the time interval studied. The study with the shortest follow-up time of 3 months (S3, Tables 3 and 4) found that pain catastrophizing predicted pain intensity but not clinicallymeaningful pain when measured as a dichotomous measure, likely reflecting power limitations. Between the two studies with 6-month follow-up that assessed pain treatment failure and presence of moderate-to-severe pain (S5 and S4, respectively), the former found a 2.7- to six-fold increased risk for high-pain catastrophizers, whereas the latter failed to observe a significant relationship. Between the two studies with $>1$ year follow-up, the larger, more methodologicallyrigorous study (S6) found an effect of catastrophizing on pain intensity, whereas the smaller unadjusted study (S2) found mixed results for an effect on non-zero pain, a divergence that likely reflects sensitivity differences in pain measures used. Thus, while length of follow-up may not interact with the strength of relation between pain catastrophizing and chronic pain outcomes, a floor effect may occur due to fewer chronic pain cases and/or general reductions in pain intensity with time.

\section{Discussion}

The objective of this systematic review was to provide a synthesis of the evidence to date on pain catastrophizing as a prospective risk factor for chronic pain (ie, persisting $\geq 3$ months) after TKA. Overall, five out of the six identified studies were able to detect some effect of pain catastrophizing on chronic pain outcomes using at least one outcome measure. Although large-scale data were not available, two mid-sized multivariate cohort studies (S3 and S6) found a positive effect of pain catastrophizing on chronic pain intensity following TKA surgery independent of baseline demographics, pain levels, and psychological factors. Whereas, two mid-sized multivariate case-control analyses (S3 and S4) failed to replicate such an effect when clinically-meaningful pain thresholds were employed as case definitions and additional adjustment was made for anxiety and depression, another multivariable case-control study found that high pain catastrophizers had dramatically increased odds of TKA-treatment failure with respect to chronic pain outcomes (S5). A lagged analysis (S1) found that pain catastrophizing remained a stable predictor of future pain severity over 1 year follow-up, and a small unadjusted (S2) study found a measurable effect of catastrophizing on non-zero pain 2 years postoperatively. These data provide a moderate level of evidence that high pain catastrophizing is a risk factor for chronic pain following TKA.

A major shortcoming in all identified studies was failure to report and adjust for analgesic use in multivariate models. It is possible that high pain catastrophizers had lower adherence to pain medications, perhaps due to heightened concerns regarding potential side-effects, which in turn may have led to poorer pain control at follow-up, thus confounding the investigated relations.

Another important consideration that remains to be resolved is whether other psychological constructs (such as anxiety and depression), which are known to be related to pain catastrophizing, were measured and included as covariates in the model predicting chronic pain. For example, the global pain rating parameter estimate for pain catastrophizing in the lagged analysis (S1) lost significance after further inclusion of depression in the model, although nighttime pain remained significant after inclusion of depression. Further, the two non-significant case-control analyses that used clinically meaningful outcomes (S3 and S4) adjusted for anxiety and depression, although their potential impact on catastrophizing estimates was not reported. These studies suggest that depression and anxiety may be more relevant exposures, and to the extent that these are causally related to 
development of chronic pain, there are well-established treatments for both. In contrast, pain catastrophizing remained an independent predictor after controlling for anxiety (S3) and/ or depression $(\mathrm{S} 3, \mathrm{~S} 4)$ in the two prospective multivariable cohort studies, which together constitute the highest level of evidence available to date. Taken together, we currently lack sufficient evidence to conclude which risk factor(s) among the many related psychological constructs (eg, pain catastrophizing, anxiety, and depression) contribute uniquely to chronic pain after TKA.

In general, pain catastrophizing levels remained stable over follow-up, whereas pain levels gradually diminished, on average (Table 4). However, length of follow-up did not appear to modify the effect of catastrophizing on chronic pain outcomes measured, with no clear pattern of significance/ non-significance observed as follow-up intervals increased. Rather, detection of a pain catastrophizing effect appeared to depend more on adequate power. Thus, the primary consideration in studies with lengthy follow-up periods (ie, $>1$ year) should be ensuring adequate numbers of cases or sufficient sensitivity in outcome measures to detect minor variability in low-grade pain. Nevertheless, without statistical analysis of large-scale data that models baseline catastrophizing on repeated pain measures over time, an interaction effect of follow-up time on the investigated relationship cannot be completely dismissed.

We observed substantial heterogeneity in studies with respect to study designs, analyses employed, multivariate adjustments, measures used, and outcome reporting. Of particular importance, each study we identified employed a different outcome measure for pain. The failure to adopt standardized outcome measures of pain intensity as well as a relevant cut-off for clinically-meaningful pain precludes direct comparison of results and limits opportunities for meta-analysis. Authors did not typically report stepwise results of multivariate models or univariate parameter estimates to aid the reader in understanding the impact of collinearity. Furthermore, the identified studies did not report specific parameter estimates or confidence intervals for non-significant findings, limiting their interpretation and further precluding meta-analysis of small sample data. For instance, although three studies used logistic regression to assess the impact of pain catastrophizing on pain outcomes (S3, S4, S5), only the study with significant results (S5) presented ORs and CIs. Assessment of exposure was more consistent, with five of six studies employing the PCS. However, the one study that dichotomized patients as high vs low pain catastrophizers (a potentially valuable clinical distinction) failed to provide data on the validity of their threshold.

To improve future data capture, the field would benefit from the adoption of the Initiative on Methods, Measurement, and Pain Assessment in Clinical Trials consensus guidelines for standardized reporting of clinically relevant pain outcomes in RCTs, which have direct relevance to both cohort and case-control studies as well. ${ }^{19,35-37}$ Multivariable adjustment should include analgesic use (type, dose), depression, and anxiety, in addition to traditional clinical risk factors. Further, investigation of the optimal PCS cut-off for 'high pain catastrophizers' in predicting adverse post-surgical pain outcomes (ROC analysis) would both set the stage for its inclusion in disease complexity measures in future investigations and would be of high clinical relevance as a practical screening tool. Finally, studies should report parameter and confidence estimates for non-significant findings so that future investigators may pool their data for powerful metaanalyses.

Strengths and limitations of our systematic review deserve comment. Strengths of our review include a comprehensive and replicable research strategy with two independent raters and quality assessment of all articles by three independent raters, as well as a systematic approach to evidence synthesis on the predictive value of catastrophizing on chronic post-TKA pain. A limitation of our review is that the identification and selection of relevant articles may have been influenced by publication bias (ie, underreporting of non-significant findings). Further, due to the heterogeneity in data capture and reporting across the included studies, a quantitative meta-analysis was not possible.

Although further well-controlled and large-scale data would be valuable, the current evidence provides moderate support that pain catastrophizing is an important risk factor for chronic pain following TKA surgery. Given that pain catastrophizing constitutes a modifiable response to threat among other populations of chronic pain patients ${ }^{38-40}$ and to the extent that it is a causal risk factor, interventions aimed at reducing pain catastrophizing symptoms ${ }^{41}$ may translate to improved pain outcomes of TKA. As rates of TKA continue to increase with the aging population and rising obesity epidemic, ${ }^{1,3}$ further clarification of the prognostic value of various pain catastrophizing levels holds promise to close the gap in TKA recovery outcomes.

\section{Acknowledgments}

Lindsay C Burns is supported by a Canadian Institutes of Health Research (CIHR) Frederick Banting and Charles 
Best Canada Graduate Scholarships Doctoral Award. Hance Clarke is supported by a Merit Award (Department of Anaesthesia, University of Toronto) and the CIHR STAGE Training Program in Genetic Epidemiology. Joel Katz is supported by a CIHR Canada Research Chair in Health Psychology at York University.

\section{Disclosure}

The authors report no conflicts of interest in this work.

\section{References}

1. Cram P, Lu X, Kates SL, Singh JA, Li Y, Wolf BR. Total knee arthroplasty volume, utilization, and outcomes among Medicare beneficiaries, 1991-2010. JAMA. 2012;308(12):1227-1236.

2. Ravi B, Croxford R, Reichmann WM, Losina E, Katz JN, Hawker GA. The changing demographics of total joint arthroplasty recipients in the United States and Ontario from 2001 to 2007. Best Pract Res Clin Rheumatol. 2012;26(5):637-647.

3. Canadian Institute of Health Information. Hip and Knee Replacements in Canada: Canadian Joint Replacement Registry 2014 Report; 2014.

4. Wylde V, Dieppe P, Hewlett S, Learmonth ID. Total knee replacement: is it really an effective procedure for all? Knee. 2007;14(6): 417-423.

5. Edwards RR, Haythornthwaite JA, Smith MT, Klick B, Katz JN. Catastrophizing and depressive symptoms as prospective predictors of outcomes following total knee replacement. Pain Res Manag. 2009;14(4):307-311.

6. Baker PN, van der Meulen JH, Lewsey J, Gregg PJ. The role of pain and function in determining patient satisfaction after total knee replacement Data from the national joint registry for England and Wales. $J$ Bone Joint Surg Br. 2007;89(7):893-900.

7. Brander VA, Stulberg SD, Adams AD, et al. Predicting total knee replacement pain: a prospective, observational study. Clin Orthop Relat Res. 2003;416:27-36.

8. Elson DW, Brenkel IJ. Predicting pain after total knee arthroplasty. J Arthroplasty. 2006;21(7):1047-1053.

9. Harden RN, Bruehl S, Stanos S, et al. Prospective examination of pain-related and psychological predictors of CRPS-like phenomena following total knee arthroplasty: a preliminary study. Pain. 2003;106(3):393-400.

10. Lingard EA, Katz JN, Wright EA, Sledge CB. Predicting the outcome of total knee arthroplasty. J Bone Joint Surg Am. 2004;86(10):2179-2186.

11. McCracken LM, Spertus IL, Janeck AS, Sinclair D, Wetzel FT. Behavioral dimensions of adjustment in persons with chronic pain: pain-related anxiety and acceptance. Pain. 1999;80(1-2):283-289.

12. Jensen MP, Moore MR, Bockow TB, Ehde DM, Engel JM. Psychosocial factors and adjustment to chronic pain in persons with physical disabilities: a systematic review. Arch Phys Med Rehabil. 2011;92(1):146-160.

13. Turk DC, Wilson HD. Fear of pain as a prognostic factor in chronic pain: conceptual models, assessment, and treatment implications. Curr Pain Headache Rep. 2010;14(2):88-95.

14. Sullivan M, Tanzer M, Stanish W, et al. Psychological determinants of problematic outcomes following total knee arthroplasty. Pain 2009;143(1-2):123-129.

15. Sullivan MJL, Bishop SR, Pivik J. The Pain Catastrophizing Scale: development and validation. Psychol Assess. 1995;7(4):524-532.

16. Granot M, Ferber SG. The roles of pain catastrophizing and anxiety in the prediction of postoperative pain intensity: a prospective study. Clin J Pain. 2005;21(5):439-445.

17. Pavlin DJ, Sullivan MJL, Freund PR, Roesen K. Catastrophizing: a risk factor for postsurgical pain. Clin J Pain. 2005;21(1):83-90.
18. Vissers MM, Bussmann JB, Verhaar JAN, Busschbach JJV, Bierma-Zeinstra SMA, Reijman M. Psychological factors affecting the outcome of total hip and knee arthroplasty: a systematic review. Semin Arthritis Rheum. 2012;41(4):576-588.

19. Farrar JT, Young JP Jr, LaMoreaux L, Werth JL, Poole RM. Clinical importance of changes in chronic pain intensity measured on an 11-point numerical pain rating scale. Pain. 2001;94(2):149-158.

20. Roth ML, Tripp DA, Harrison MH, Sullivan M, Carson P. Demographic and psychosocial predictors of acute perioperative pain for total knee arthroplasty. Pain Res Manag. 2007;12(3 Autumn):185-194.

21. International Association for the Study of Pain Task Force on Taxonomy. Classification of Chronic Pain: Descriptions of Chronic Pain Syndromes and Definitions of Pain Terms. Seattle: IASP Press; 1994.

22. Yusuf E, Nelissen RG, Ioan-Facsinay A, et al. Association between weight or body mass index and hand osteoarthritis: a systematic review. Ann Rheum Dis. 2010;69(4):761-765.

23. Olthof M, Stevens M, Bulstra SK, van den Akker-Scheek I. The association between comorbidity and length of hospital stay and costs in total hip arthroplasty patients: a systematic review. J Arthroplasty. 2014;29(5):1009-1014.

24. Forsythe ME, Dunbar MJ, Hennigar AW, Sullivan MJ, Gross M Prospective relation between catastrophizing and residual pain following knee arthroplasty: two-year follow-up. Pain Res Manag. 2008; 13(4):335-341.

25. Masselin-Dubois A, Attal N, Fletcher D, et al. Are psychological predictors of chronic postsurgical pain dependent on the surgical model? A comparison of total knee arthroplasty and breast surgery for cancer. $J$ Pain. 2013;14(8):854-864.

26. Noiseux NO, Callaghan JJ, Clark CR, Zimmerman MB, Sluka KA, Rakel BA. Preoperative predictors of pain following total knee arthroplasty. J Arthroplasty. 2014;29(7):1383-1387.

27. Riddle DL, Wade JB, Jiranek WA, Kong X. Preoperative pain catastrophizing predicts pain outcome after knee arthroplasty. Clin Orthop Relat Res. 2010;468(3):798-806.

28. Sullivan M, Tanzer M, Reardon G, Amirault D, Dunbar M, Stanish W. The role of presurgical expectancies in predicting pain and function one year following total knee arthroplasty. Pain. 2011;152(10):2287-2293.

29. Rosenstiel AK, Keefe FJ. The use of coping strategies in chronic low back pain patients: relationship to patient characteristics and current adjustment. Pain. 1983;17(1):33-44.

30. Melzack R. The short-form McGill Pain Questionnaire. Pain. 1987;30(2):191-197.

31. Cleeland CS. Measurement of pain by subjective report. In: Chapman CR, Leser JD, editors. Issues in Pain Management. Vol 12. New York: Raven Press; 1989:391-403.

32. Bellamy N, Buchanan WW, Goldsmith CH, Campbell J, Stitt LW. Validation study of WOMAC: a health status instrument for measuring clinically important patient relevant outcomes to antirheumatic drug therapy in patients with osteoarthritis of the hip or knee. J Rheumatol. 1988;15(12):1833-1840.

33. Katz J, Melzack R. Measurement of pain. Surg Clin North Am. 1999; 79(2):231-252.

34. Sullivan MJL. The Pain Catastrophizing Scale: User Manual. Montreal: McGill University; 2009:1-36.

35. Turk DC, Dworkin RH, Allen RR, et al. Core outcome domains for chronic pain clinical trials: IMMPACT recommendations. Pain. 2003; 106(3):337-345.

36. Dworkin RH, Turk DC, Farrar JT, et al. Core outcome measures for chronic pain clinical trials: IMMPACT recommendations. Pain. 2005; 113(1-2):9-19.

37. Farrar JT, Portenoy RK, Berlin JA, Kinman JL, Strom BL. Defining the clinically important difference in pain outcome measures. Pain. 2000; 88(3):287-294.

38. Wertli MM, Burgstaller JM, Weiser S, Steurer J, Kofmehl R, Held U. Influence of catastrophizing on treatment outcome in patients with nonspecific low back pain: a systematic review. Spine. 2014; 39(3):263-273. 
39. Gallagher L, McAuley J, Moseley GL. A randomized-controlled trial of using a book of metaphors to reconceptualize pain and decrease catastrophizing in people with chronic pain. Clin J Pain. 2013;29(1):20-25.

40. Smeets RJ, Vlaeyen JW, Kester AD, Knottnerus JA. Reduction of pain catastrophizing mediates the outcome of both physical and cognitive-behavioral treatment in chronic low back pain. J Pain. 2006; 7(4):261-271.
41. Clarke H, Kirkham KR, Orser BA, et al. Gabapentin reduces preoperative anxiety and pain catastrophizing in highly anxious patients prior to major surgery: a blinded randomized placebo-controlled trial. Can J Anaesth. 2013;60(5):432-443.

Journal of Pain Research

\section{Publish your work in this journal}

The Journal of Pain Research is an international, peer-reviewed, open access, online journal that welcomes laboratory and clinical findings in the fields of pain research and the prevention and management of pain. Original research, reviews, symposium reports, hypothesis formation and commentaries are all considered for publication.

The manuscript management system is completely online and includes a very quick and fair peer-review system, which is all easy to use. Visit http://www.dovepress.com/testimonials.php to read real quotes from published authors. 